\title{
Novel MR-Guided Radiotherapy Elective Rotation for Radiation Oncology Trainees
}

\author{
Austin J. Sim ${ }^{1}$, Jessica M. Frakes ${ }^{1}$, Sarah E. Hoffe ${ }^{1}$, Evan Wuthrick ${ }^{1}$, Thomas J. Dilling ${ }^{1}$, Stephen \\ Rosenberg ${ }^{1}$ \\ 1. Radiation Oncology, Moffitt Cancer Center, Tampa, USA
}

Corresponding author: Stephen Rosenberg, stephen.rosenberg@moffitt.org

\begin{abstract}
MR-guided adaptive radiation therapy (RT) is emerging as an integral treatment modality for certain applications and is poised to become an exciting opportunity for greater treatment precision and personalization. However, this is still a relatively nascent technology and only a few institutions and programs have access to this technology for clinical use and trainee education. To increase the diversity of elective offerings and improve the understanding of an MR-guided radiotherapy program, we initiated a unique MR-guided radiotherapy elective rotation for radiation oncology residents. During a representative four-week rotation, 21 simulations were completed by the resident on service. A plurality of simulations were for pancreas stereotactic body radiation therapy (SBRT; $48 \%$ ) and a majority ( $71 \%$ ) of simulations were for adaptive treatments. Additionally, 74 adaptive fractions were completed during this month, of which a significant majority (74\%) were for pancreas SBRT. Of the non-adaptive fractions, the majority were for prostate SBRT and intensity-modulated radiation therapy (IMRT). Although many programs may offer training in some aspects of MR-guided radiotherapy as trainees rotate through certain disease sites, we hope this may serve as a blueprint to encourage programs with this technology to fully embrace training in essential competencies related to MR-guided radiotherapy. MR-guided radiotherapy has unique challenges that trainees need to understand to deliver treatment safely: geometric uncertainty, MRI to RT isocenter, and uncertainties with voxel size/tracking.
\end{abstract}

Review began 09/18/2020 Review ended 09/27/2020 Published 09/29/2020

(c) Copyright 2020

Sim et al. This is an open access article distributed under the terms of the Creative Commons Attribution License CC-BY 4.0., which permits unrestricted use, distribution, and reproduction in any medium, provided the original author and source are credited.
Categories: Medical Education, Radiation Oncology, Healthcare Technology

Keywords: magnetic resonance imaging-guided radiation therapy (mrigrt), stereotactic body radiation therapy (sbrt), adaptive radiation therapy, graduate medical education

\section{Introduction}

MR-guided adaptive radiation therapy (RT) is emerging as an integral treatment modality for certain applications and is poised to become an exciting opportunity for greater treatment precision and personalization. The improved soft tissue contrast and opportunities for real-time, intrafraction tracking allow for the reduction of planning target volume (PTV) by decreasing tumor and organ at risk uncertainty [1]. The emergence of radiomic biomarkers, use of multiple imaging sequences, offers unique opportunities to see day-to-day changes in radiomic features to develop novel biomarkers to assess for tumor response, as has already started in diagnostic functional imaging [2]. Two commercial systems are FDA approved and are in routine clinical use in the United States, with many more systems under development across the globe [3]. However, this is still a relatively nascent technology and only a few institutions and programs have access to this technology for clinical use and trainee education. We have previously examined a lack of MRI familiarity among trainees in radiation oncology [4]. In that context, we have worked to develop an MR-guided elective to improve the understanding of this technology for residents in our program.

An MR-linac has been in routine clinical use at our institution (Moffitt Cancer Center, Tampa, FL) since February 2019. We have completed over 250 MR-guided simulations in those 16 months with approximately one-third of patients being treated with adaptive radiotherapy. On the MR-linac at our institution, the breakdown by treatment of disease site is $30 \%$, pancreas; $20 \%$, thoracic; $15 \%$, prostate; $10 \%$, liver, and mixture of other sites.

In the United States, residency in radiation oncology lasts for four years after completion of an intern year. After the completion of required rotations and up to 12 months of elective/research time, residents in our program typically have two or three additional months of clinical elective time. Residents are able to take this time to revisit disease sites or pursue new ones, like cutaneous. To increase the diversity of elective offerings and improve the understanding of MR-guided radiotherapy program, we initiated a unique MRguided radiotherapy elective rotation for radiation oncology residents.

\section{Materials And Methods}

Data regarding number and types of simulations and adaptive treatments were collected for a single resident on a representative four-week rotation. Results are described descriptively. 


\section{Cureus}

\section{Results}

This four-week rotation is designed for senior residents in their penultimate or final years (postgraduate year [PGY]-4 and PGY-5) who have completed rotations in core disease sites. During this elective, the resident covers all simulations on our MR-linac not already covered by another service and all adaptive fractions. Coverage of non-adaptive stereotactic body radiation therapy (SBRT) fractions is encouraged but not mandatory. Contours are completed by the resident and reviewed with the treating attending physician. During adaptive fractions, the resident is primarily responsible for confirming rigid shifts, recontouring critical structures based on the anatomy of the day, evaluating adaptive plans, and adapted plan quality assurance, with final plan approval by the attending physician and medical physicist.

During a representative four-week rotation, all simulations not covered by another resident were completed by the resident on service ( $\mathrm{n}=21$, an additional seven MR-guided prostate SBRT simulations were completed by the resident on the genitourinary service). A plurality of simulations were for pancreas SBRT (48\%) and $71 \%$ of simulations were for adaptive treatments (Figures $1 a, 1 b$ ). Seventy-four adaptive fractions were completed during this month, of which a significant majority (74\%) were for pancreas SBRT. Of the nonadaptive fractions, the majority were for prostate SBRT and intensity-modulated radiation therapy (IMRT) (Figures 2a, 2b).

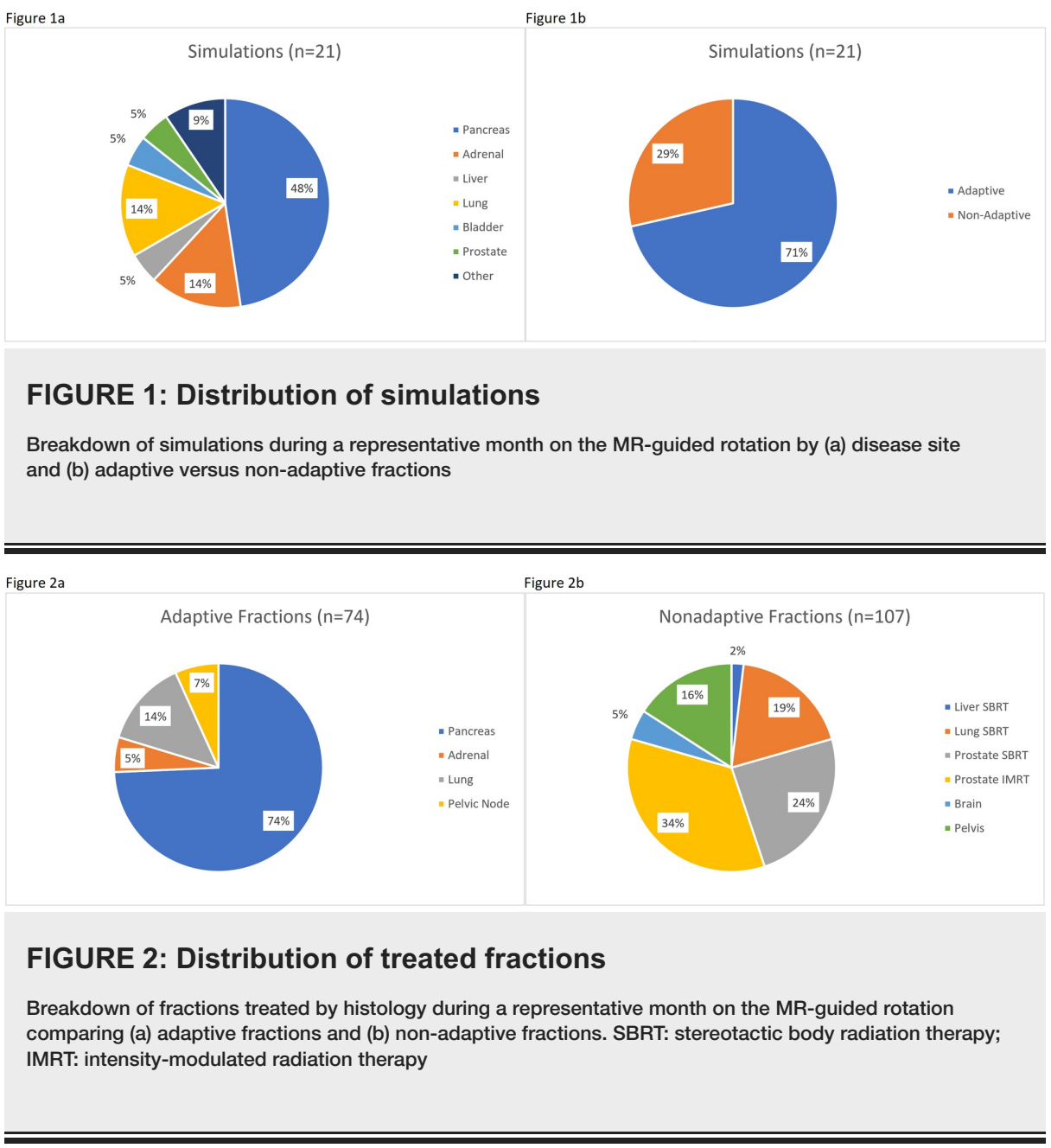

Expected competencies to be mastered by the trainee include (1) the indications and appropriate patient selection for online and offline MR-guided radiotherapy, (2) unique considerations of tracking structures at patient simulation, and (3) real-time re-planning for online adaptive radiotherapy (Table 1). 


\section{Cureus}

\section{PGY-4 objectives}

PGY-5 objectives

Competency: Medical knowledge

Goal: Clinical knowledge

- Be able to determine which patients are eligible or ineligible for MR-guided radiotherapy.

- Fully understand indications for MR-guided radiotherapy including rarer entities across anatomical sites.

- Fully understand the indications for real-time (online) and offline adaptive MR-guided radiotherapy.

- Understand treatment results and outcomes of MR-guided radiotherapy across malignancies.

- Fully understand advantageous and disadvantages to MRguided radiotherapy.

Goal: Medical physics knowledge

- Apply concepts of medical physics as it relates to MR-guided radiotherapy.

- Understand and be able to describe TRUFI sequence and basic MRI physics.

- Understand and describe the different re-optimization processes and QA process as part of real-time adaptive MRguided radiotherapy.

Competency: Patient care

Goal: Patient care

- Understand decisions on ROI and tracking as it relates to MRguided radiation.

- Understand the need for CT scans as part of MR-guided treatment and when CT scans may not be necessary.

- Delineate all target volumes nearly independently (GTV, CTV, PTV) on MRI and CT.

- Perform the real-time review of patient treatment plans as it pertains to adaptive MR-guided treatment and delivery (i.e., delivery of original plan or adaptive plan).

- Plan and perform MR-guided radiotherapy with minimal faculty member assistance
- Understand treatment results and outcomes of MR-guided radiotherapy and be able to describe clinical trials (results and ongoing trials) and retrospective series.

- Master acute and chronic effects of MR-guided radiation therapy across disease sites.

- Master delivery of real-time adaptive MR-guided radiotherapy (online and offline) across disease sites.
- Thoroughly understand medical physics concepts for safe delivery of MR-guided radiation therapy.
- Make independent decisions on $\mathrm{ROI}$ and tracking structures for MR-guided treatment.

- Master the real-time review of patient treatment plans as it pertains to adaptive MR-guided treatment and delivery (i.e., delivery of original plan or adaptive plan).

\section{TABLE 1: MR-guided radiotherapy elective objectives}

PGY: postgraduate year; TRUFI: true fast imaging with steady-state precession; QA: quality assurance; ROI: region of interest; GTV: gross tumour volume; CTV: clinical target volume; PTV: planning target volume

\section{Discussion}

To our knowledge, this is the first reported MR-guided radiotherapy elective rotation to be offered for radiation oncology trainees. Comfort with MR-guidance and adaptive radiotherapy is becoming a soughtafter skill, especially in the treatment of certain thoracic [5] and gastrointestinal malignancies [6]. The use of diagnostic MR imaging in staging, treatment planning, and surveillance is rapidly becoming integral to the standard of care of an increasing number of disease sites, necessitating a shift in resident education to 
inculcate facility in interpreting and using these images. Additionally, online adaptive radiotherapy presents new skills for trainees to master [7]. Much like the chief resident service described by Jeans et al. [8], we believe this unique curricular offering addresses a need as we look to continually modernize our educational curricula to account for new technologies.

Although many programs may offer training in some aspects of MR-guided radiotherapy as trainees rotate through certain disease sites, we hope this may serve as a blueprint to encourage programs with this technology to fully embrace training in essential competencies related to MR-guided radiotherapy. MRguided radiotherapy has unique challenges that trainees need to understand to deliver treatment safely: geometric uncertainty, MRI to RT isocenter, and uncertainties with voxel size/tracking. These were reviewed throughout the rotation, which gives trainees the tools to develop confidence in understanding how to troubleshoot difficult cases and safety in MR-guided treatment.

\section{Conclusions}

As MR-guided radiotherapy continues to expand throughout the United States and worldwide, it is poised to become an essential tool in the radiotherapy armamentarium. As efforts are underway to reform the educational curriculum in radiation oncology, inclusion of MR-guided and adaptive radiotherapy should be considered more formally in radiation oncology residency training.

\section{Additional Information}

\section{Disclosures}

Human subjects: All authors have confirmed that this study did not involve human participants or tissue. Animal subjects: All authors have confirmed that this study did not involve animal subjects or tissue. Conflicts of interest: In compliance with the ICMJE uniform disclosure form, all authors declare the following: Payment/services info: All authors have declared that no financial support was received from any organization for the submitted work. Financial relationships: Thomas J. Dilling declare(s) personal fees and non-financial support from Harborside Press. Outside of submitted work. Thomas J. Dilling declare(s) personal fees from Varian. Outside of submitted work. Thomas J. Dilling declare(s) personal fees and non-financial support from National Comprehensive Cancer Network. Outside of submitted work. Stephen A. Rosenberg declare(s) personal fees from Novocure. Outside of submitted work. Thomas J. Dilling declare(s) non-financial support from AstraZeneca. Outside of submitted work. Other relationships: All authors have declared that there are no other relationships or activities that could appear to have influenced the submitted work.

\section{References}

1. Hall WA, Paulson ES, van der Heide UA, et al.: The transformation of radiation oncology using real-time magnetic resonance guidance: a review. Eur J Cancer. 2019, 122:42-52. 10.1016/j.ejca.2019.07.021

2. Kiser KJ, Smith BD, Wang J, Fuller $\mathrm{CD}$ : "Après Mois, Le Déluge": preparing for the coming data flood in the MRI-guided radiotherapy era. Front Oncol. 2019, 9:983. 10.3389/fonc.2019.00983

3. Das IJ, McGee KP, Tyagi N, Wang H: Role and future of MRI in radiation oncology . Br J Radiol. 2019, 91:20180505. 10.1259/bjr.20180505

4. Singer L, Rosenberg SA: The impact of MRI on radiation oncology graduate medical education . J Am Coll Radiol. 2019, 16:859-863. 10.1016/j.jacr.2018.11.030

5. Finazzi T, Haasbeek CJA, Spoelstra FOB, et al.: Clinical outcomes of stereotactic MR-guided adaptive radiation therapy for high-risk lung tumors. Int J Radiat Oncol Biol Phys. 2020, 107:270-278. 10.1016/j.ijrobp.2020.02.025

6. Rudra S, Jiang N, Rosenberg SA, et al.: Using adaptive magnetic resonance image-guided radiation therapy for treatment of inoperable pancreatic cancer. Cancer Med. 2019, 8:2123-2132. 10.1002/cam4.2100

7. Green OL, Henke LE, Hugo GD: Practical clinical workflows for online and offline adaptive radiation therapy. Semin Radiat Oncol. 2019, 29:219-227. 10.1016/j.semradonc.2019.02.004

8. Jeans EB, Beard TB, Boon AL, et al.: Empowering residents into independent practice: a single-institutional endeavor aimed at developing resident autonomy through implementation of a chief resident service in radiation oncology. Int J Radiat Oncol Biol Phys. 2020, 107:23-26. 10.1016/j.ijrobp.2020.01.001 